\title{
User adaptation to closed-loop decoding of motor imagery termination
}

\author{
Bastien Orset, Kyuhwa Lee, Ricardo Chavarriaga, Senior Member, IEEE and José del R. Millán, Fellow, IEEE
}

\begin{abstract}
One of the most popular methods in non-invasive brain machine interfaces (BMI) relies on the decoding of sensorimotor rhythms associated to sustained motor imagery. Although motor imagery has been intensively studied, its termination is mostly neglected. Objective: Here, we provide insights in the decoding of motor imagery termination and investigate the use of such decoder in closed-loop BMI. Methods: Participants $(\mathbf{N}=9)$ were asked to perform kinesthetic motor imagery of both hands simultaneously cued with a clock indicating the initiation and termination of the action. Using electroencephalogram (EEG) signals, we built a decoder to detect the transition between event-related desynchronization and event-related synchronization. Features for this decoder were correlates of motor termination in the upper $\mu$ and $\beta$ bands. Results: The decoder reached an accuracy of $76.2 \%$ $(\mathbf{N}=9)$, revealing the high robustness of our approach. More importantly, this paper shows that the decoding of motor termination has an intrinsic latency mainly due to the delayed appearance of its correlates. Because the latency was consistent and thus predictable, users were able to compensate it after training. Conclusion: Using our decoding system, BMI users were able to adapt their behavior and modulate their sensorimotor rhythm to stop the device (clock) accurately on time. Significance: These results show the importance of closed-loop evaluations of BMI decoders and open new possibilities for BMI control using decoding of movement termination.
\end{abstract}

Index Terms - Brain-machine interface (BMI), EEG, motor imagery, motor termination, latency, decoding adaptation.

\section{INTRODUCTION}

$\mathrm{B}$ rain machine interfaces (BMIs) aim at providing communication and control pathways for people with motor disabilities [1]. A BMI bypasses the natural motor pathways, enabling users to control a large variety of external devices and interact directly with their environment, as the BMI decodes users' intentions directly from the analysis of brain signals and translates intentions into commands for an external device such as spellers [2], [3], avatars [4], robots and wheelchairs [5], [6], hand neuroprostheses [7], [8] as well as for neurogaming and consciousness assessment [9], [10].

This paper was submitted on the $22^{\text {nd }}$ February 2019. A complementary analysis of the data recorded during the offline session reported here appeared in a conference paper [11]. This work was supported by the Swiss NCCR Robotics. B. O., K. L., R. C. are with Ecole Polytechnique Fédérale de Lausanne, Geneva, Switzerland (email: bastien.orset@epfl.ch). J. d. R. M. is with the Dept. of Electrical and Computer Engineering \& the Dept. of Neurology, University of Texas at Austin, USA; he is also with Ecole Polytechnique Fédérale de Lausanne (email: jose.millan@austin.utexas.edu). R. C is also with ZHAW Datalab, Zurich University of Applied Sciences, Winterthur, Switzerland.
Most non-invasive BMIs based on voluntary modulations of brain rhythms aim at detecting the initiation of an imagined movement. Hence decoders are usually trained on samples from the periods before and after onset. Once the onset is detected, predefined commands can be triggered. Although the detection of imagined movement initiation is critical in the process, decoding the volitional interruption of motor imagery (MI) is of equal importance in order to endow brain-actuated devices with more natural behavior. While decoding of movement initiation is the focus of multiple works [7], [8], [12], [13], decoding of movement termination has been rarely investigated [14], [15]. Indeed, only two studies have explored so far the feasibility of decoding termination of MI. The first one showed the possibility to build a brain switch using one Laplacian channel [16]. Similarly, Bai et al. [17] investigated a $\beta$ rhythm-based $\mathrm{BMI}$ in repetitive motor imagery.

In this study, we investigate the use of a specific decoder for hand MI termination in a closed-loop BMI. We show for the first time that, during closed-loop operation, BMI users can adapt to their own decoder and compensate for its latency to stop precisely on time. To this end, we designed a task enabling us to capture the correlates of sustained MI as well as the neural correlates of MI termination. This task was inspired by Libet's experiment on motor initiation [18] and explored in the context of BCI [19].

It is known that changes in the brain rhythms during planning and execution of movements, as well as in the case of MI, can be observed in and decoded from human EEG [20]. After movement termination, an increase of power (event-related synchronization, ERS) is induced in the $\beta$ band $(13-25 \mathrm{~Hz})$. Such synchronization, often called $\beta$ rebound, can last for about a second. Although the role of $\beta$ rebound is still under debate, it is currently thought to have a function of inhibition of the motor cortex by somatosensory processing [21], [22]. Oscillatory activity in the $\beta$ band has been also linked to an active process aiming to maintain the current sensorimotor or cognitive state (i.e., status quo) [23]. Similarly, it has also been reported the presence of an ERS in the $\mu$ band $(8-13 \mathrm{~Hz})$ that could be interpreted as an electrophysiological correlate of cortical idling state in sensorimotor areas [24]-[26]. Such synchronizations can be explained by an increase of rhythmic activity paradoxically due to a decrease of excitability of cortical neurons or inhibited cortical neurons [27], [28]. This was also reported after MI tasks [28]. When performing handrelated motor tasks, ERS can mainly be observed in the contralateral hand representation area. In the $\beta$ band, this synchronization can also be seen in the supplementary motor area (SMA) located in mid-central areas of the brain with 
slightly higher frequencies and an earlier beginning compared to the contralateral ERS [29], [30].

\section{METHODS}

\section{A. Experiment setup}

Nine healthy naïve subjects (19-26 years, 2 females) participated in the experiment. The study was approved by the Cantonal Committee of Vaud, Switzerland for ethics in human research (CER-VD) and subjects gave their written permission and signed a consent form.

\section{B. Offline Protocol}

Participants were comfortably seated in front of a PC monitor and asked to perform kinesthetic MI (i.e., imagining the feeling associated with performing a movement) of both hands simultaneously. The total duration of a trial was $13 \mathrm{~s}$. Subjects first fixated the cross in the middle of the screen $(3 \mathrm{~s})$, then they were instructed to performed MI once the clock hand was moving ( 2 to $4 \mathrm{~s}$ ), and finally were asked to stop their MI and stay calm until the hand clock finishes its turn (6 to $8 \mathrm{~s})$. The total time of a clock hand revolution (MI plus rest) was $10 \mathrm{~s}$. During the rest period after MI termination (MIt), subjects were instructed to stay calm avoiding any muscular contraction or blinks while the hand finished to revolve around the clock. In between trials, a relaxation phase of $7 \mathrm{~s}$ was introduced to allow participants to blink and rest. Fig. 1 illustrates the structure of the trial. Each subject performed 4 runs of 30 trials each (120 trials in total).

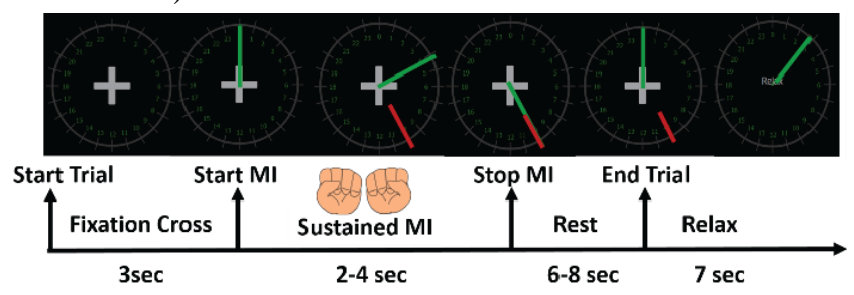

Fig. 1. Trial structure during offline protocol. During a trial, the subject is asked to continuously look at a fixation cross in the center of the clock. The subject is instructed to stay calm for the first 3 seconds without moving or blinking. A clock hand (green bar) indicates to the subject to initiate his motor imagery of both hands. When the clock hand reaches a target (red bar), the subject stops motor imagery and stays at rest (no blink or movement). A period of 7 seconds following each trial allows the subject to relax.

\section{Online Protocol}

After training a decoder on the offline data (c.f. Section II-E), participants' task was to stop the clock hand on a target in realtime by terminating their MI action. Participants were asked to adapt to their individual decoder in order to stop precisely on the target. To do so, participants had to evaluate the latency of the BMI output during an initial calibration phase that preceded the actual experiment. The calibration phase consisted of 20 online trials and, afterwards, each subject performed 4 runs of 25 trials each (100 trials in total).

In this protocol, a gauge (not shown in Fig.1) was additionally integrated on the clock hand showing the BMI output as a source of continuous feedback. The clock hand stopped when the gauge was filled $\left(\mathrm{P}_{\text {gauge, }}=1\right)$. The BMI output corresponded to the integration of the output probabilities of the MIt decoder to each single EEG sample based on an exponential moving average (Eq.1)[31]:

$$
\mathrm{P}_{\mathrm{t}}=\alpha \mathrm{P}_{\mathrm{t}-1}+(1-\alpha) \mathrm{p}_{\mathrm{t}}
$$

Where $\mathrm{P}_{\mathrm{t}}$ is the smoothed likelihood, $\mathrm{p}_{\mathrm{t}}$ is the likelihood of detecting MI termination (output of the MIt decoder) and $\alpha$ is a smoothing parameter, $0 \leq \alpha \leq 1$.

The smoothing parameter $\alpha$ was individually set for each subject by the operator during the calibration phase. The value of $\alpha$ was manually tuned so as to optimize correct delivery, increasing it in the case of substantial early delivery over the last few trials or decreasing it in the case of long late delivery. At the end of the calibration phase, the value of the smoothing parameter $\alpha$ was kept fixed for the entire online evaluation. Table 1 reports the value of this parameter for each subject.

Table 1. Individual values of the smoothing parameter $\alpha$ used during the online session. The posterior probabilities of the decoder were smoothed using exponential moving average based on equation 1 with $\alpha$ parameter set for every subject during a calibration phase of 20 trials.

\begin{tabular}{||l||l||l||l||l||l||l||l||l||l||}
\hline \hline Subject & S1 & S2 & S3 & S4 & S5 & S6 & S7 & S8 & S9 \\
\hline \hline$\alpha$ & 0.9 & 0.75 & 0.8 & 0.65 & 0.8 & 0.65 & 0.7 & 0.8 & 0.8 \\
\hline \hline
\end{tabular}

To decrease the delay due to the exponential moving average, the smoothed probabilities were passed through a burst framework which was gradually increasing the bar shown to the user. This burst framework was defined by the following equation (Eq. 2).

$$
\begin{gathered}
P_{\text {gauge }, t}=P_{\text {gauge }, t-1}+\left(P_{t}-0.5\right) \\
P_{\text {gauge }, t} \leq 1 \\
P_{\text {gauge }, t=0}=0.1
\end{gathered}
$$

\section{Recording System}

EEG signals were recorded at a sampling frequency of $512 \mathrm{~Hz}$ with 16 active surface electrodes placed over the sensorimotor cortex i.e., on positions $\mathrm{Fz}, \mathrm{FC} 3, \mathrm{FC} 1, \mathrm{FCz}, \mathrm{FC} 2$, $\mathrm{FC} 4, \mathrm{C} 3, \mathrm{C} 1, \mathrm{Cz}, \mathrm{C} 2, \mathrm{C} 4, \mathrm{CP} 3, \mathrm{CP} 1, \mathrm{CPz}, \mathrm{CP} 2$, and $\mathrm{CP} 4$ according to the international 10/10 system (reference: left earlobe; ground: AFz; g.tec gUSBamp, Guger Technologies OG, Graz, Austria).The amplifier was set with a hardware band-pass frequency between 0.01 and $100 \mathrm{~Hz}$ (Butterworth $4^{\text {th }}$ order) and a notch filter between $48 \mathrm{~Hz}$ and $52 \mathrm{~Hz}$. A common average reference was used on the EEG raw data to enhance the signal-to-noise ratio.

\section{E. Offline Classification}

Power spectral densities (PSD) were computed in a 1swindow using the Welch's method $(0.5 \mathrm{~s}$ window with $0.25 \mathrm{~s}$ non-overlapping window) from 4 to $40 \mathrm{~Hz}$ with a $2 \mathrm{~Hz}$ resolution on the 16 channels, yielding a total of 304 features. We compared three different classifiers based on different bands: $\mu$ band $(8-13 \mathrm{~Hz}), \beta$ band $(20-26 \mathrm{~Hz})$ and all bands together $(4-40 \mathrm{~Hz})$. Then for each of these classifiers, 10 -fold trial-based cross-validation was performed where the 6 best features from each fold were selected based on their Fisher Score. Using these features, a Diagonal Linear Discriminant Analysis (DLDA) was trained. Feature vectors were extracted from each sample in the training dataset and z-score normalized. Their mean and variance were applied on the feature vectors in the testing dataset. The movement termination decoders were trained to distinguish between sustained MI and MIt. 1-second long samples were computed 
with a sliding window (shifted every $62.5 \mathrm{~ms}$ ) in the time intervals $[-2,0] \mathrm{s}(\mathrm{MI})$ and $[0.5,2.5] \mathrm{s}$ (MIt) with respect to the offset $(\mathrm{t}=0)$. Using the $1 \mathrm{~s}-$ long overlapping sliding windows every $62.5 \mathrm{~ms}$ within the $2 \mathrm{~s}$ interval yield 17 samples per trial.

To assess the classification performance, we calculated the accuracy at the sample level over the 10-fold cross validation. The accuracy was defined as the number of correctly classified samples over the total number of samples and was computed for each fold. We estimated the significance threshold above chance level at the $95 \%$ confidence interval based on the inverse binomial cumulative distribution (uniform priors, $\mathrm{n}=$ 408 samples in test set), leading to a value of $54.17 \%$.

\section{F. Asynchronous classification in online session}

Based on the results of the cross-validation, and using all the data from the offline session, we trained a decoder using all the 304 features available $(4-40 \mathrm{~Hz})$. After normalization, a total of 6 features were selected based on their Fisher Score ranking. Then, these features were used to train a DLDA classifier to detect MIt in real-time during the online session. During this session, a $1 \mathrm{~s}$ buffer was used and updated every $62.5 \mathrm{~ms}$. For each buffer, PSDs were z-scored using the mean and the standard deviation found when training the decoder.

\section{G. Pseudo-online analysis}

A pseudo-online (PO) analysis was performed on the offline and online data to further study the classifier behavior in realtime at the trial level. To do so, we compare the behavior of the online classifier (online PO) and the behavior of the different classifiers used for cross-validation (offline PO). In this analysis, the classifier was tested in the time interval $[-3,4] \mathrm{s}$ with respect to the offset cue. During this time interval, the likelihood (i.e., the probability of detecting MIt) was calculated from the decoder on samples computed with a 1s-window shifted every $62.5 \mathrm{~ms}$. The posterior probabilities were then smoothed as explained in Section II.C. Using this decoder, we measured the decoding latency, which we defined as the time when the average posterior probabilities over trials were crossing the significance threshold above chance level of $54.17 \%$.

\section{RESULTS}

\section{A. Time frequency analysis}

A spectral analysis was first performed on central channels $(\mathrm{C} 3, \mathrm{Cz}, \mathrm{C} 4)$ in order to evaluate the event-related spectral changes for MI onset and offset using a Fast Fourier Transform on 1s-Hanning window shifted every $62.5 \mathrm{~ms}$ [32]. A baseline period [-2 -1] $\mathrm{s}$ with respect to the onset was used to compute the spectral changes. Fig. 2A shows the grand averages across subjects recorded during the offline session. During sustained MI, a desynchronization (ERD) could be observed in the $\mu$ and $\beta$ bands, more prominently on centro-lateral channels ( $\mathrm{C} 3, \mathrm{C} 4)$ as expected in the case of bilateral hand MI. On the opposite, an ERS was seen on these channels after stopping MI, mainly noticed in the upper $\mu$ (11-13 Hz). An ERS was also observed in the high $\beta$ band $(20-30 \mathrm{~Hz})$ mainly in $\mathrm{C} 3$ and $\mathrm{C} 4$, but it exhibited larger inter-subject variability. Importantly, one can observe that $\beta$ ERS had a shorter duration than $\mu$ ERS (Fig. 2B). Indeed, $\beta$ ERS started at about $0.8 \mathrm{~s}$ after the offset and lasted for $2.1 \mathrm{~s}$, while $\mu$ ERS started later at $1.5 \mathrm{~s}$ but remained until the end of the trial $(\alpha=0.05$, repeated measure t-tests based on t-statistics, FDR corrected for multiple comparisons).
A

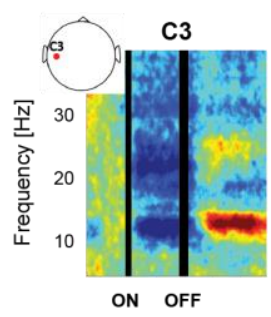

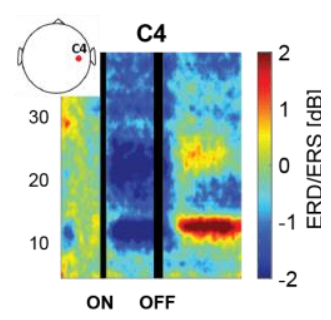

B

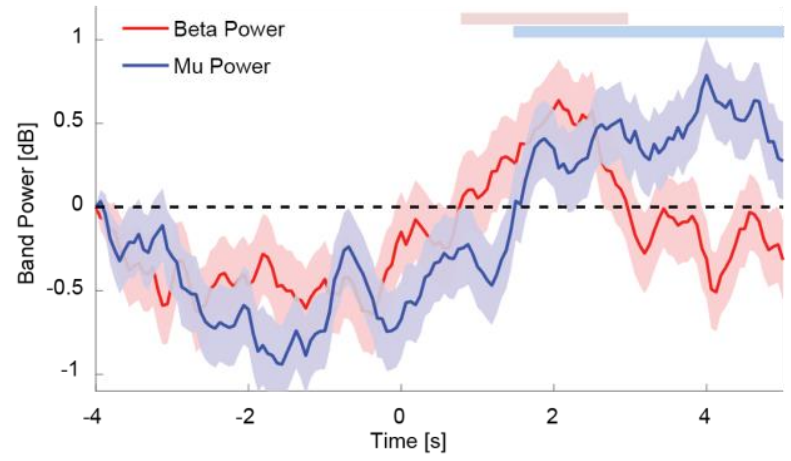

C

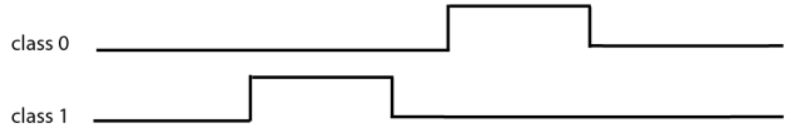

Fig. 2. A. Grand average spectrogram on central channels across subjects and averaged over all trials during offline session. The first vertical line corresponds to MI onset (ON), while the second one is MI offset (OFF). The figure has been interpolated due to the variation on MI duration. The pre and during MI period $[-34] \mathrm{s}$ was cropped and joined with the post MI period [0 6] s. ERD/ERS were computed with a logarithmic scale using a baseline period [-2 -1] s. B. Band power of the $\mu$ and $\beta$ rhythms averaged over $\mathrm{C} 3$ and $\mathrm{C} 4$ channels for all the trials. The time origin $(\mathrm{t}=0)$ corresponds to the offset. Each color bar on top indicates periods of significant difference with respect to the baseline period ([3 -2] s from MI onset) for its respective band ( $\alpha=0.05$, repeated measure t-tests based on t-statistics, FDR corrected for multiple comparisons). C. Time intervals used for classification: [-2,0] s (MI, class 0) and [0.5, 2.5] s (MI termination, class 1$)$ with respect to the offset $(\mathrm{t}=0)$.

\section{B. Offline classification}

We evaluated the performance of our classifier for decoding MI termination with three different bands: $\mu(8-13 \mathrm{~Hz}), \beta(20-$ $26 \mathrm{~Hz}$ ) and all bands $(4-40 \mathrm{~Hz})$. Fig. 3A shows the accuracy of the three decoders for each subject and in grand average. The $\mu$ and $\beta$ bands-based decoders yielded accuracies of $73.3 \pm 7.4 \%$ and $73.0 \pm 7.0 \%$ (mean \pm std), respectively; while using all bands reached an accuracy of $76.2 \pm 6.4 \%$. This performance improvement using the classifier based on all the bands was, however, not statistically significant on average (one-way ANOVA $\mathrm{F}(2,24)=0.57 ; \mathrm{p}=0.574)$. Importantly, all three classifiers reached high accuracy and were statistically above the significance threshold (54.17\%) for every subject. Additionally, Fig. 3B shows the Fisher scores of the spectral features averaged over the different folds of the cross validation and subjects in the case of the third classifier. Note that most of the discriminant features can be found in channels located over the hand motor area $(\mathrm{C} 4, \mathrm{C} 3$ and $\mathrm{CP} 4)$ in the upper $\mu$ band and, 
to a lesser extent, in the $\beta$ band. Later, we used this classifier for the online session.
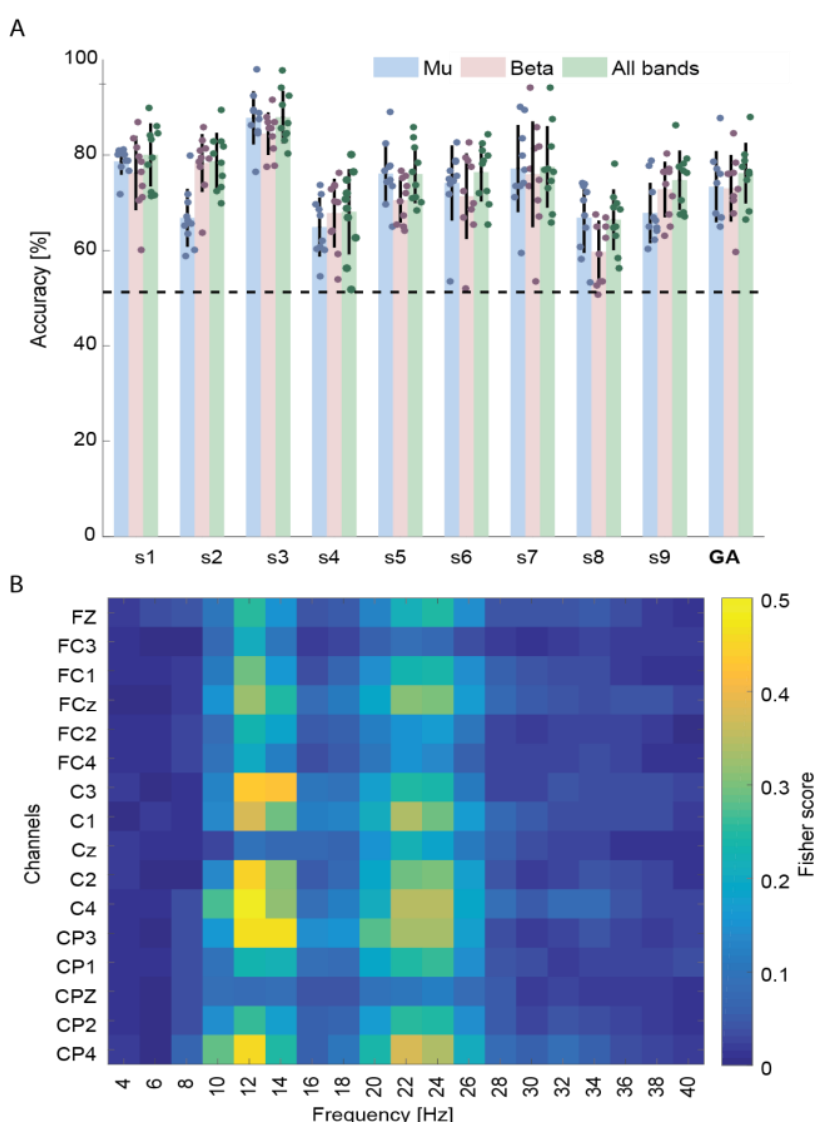

Fig. 3. Movement termination decoder. A. Classifier accuracies are reported for every subject (s1-s9) as well as in grand average (GA). The standard deviation is shown on each bar. Dots indicate performance for each subject. The horizontal dashed line corresponds to the significance threshold above chance level $(54.17 \%)$. B. Fisher score map averaged over all subjects. Fisher scores are shown for the features (channels $x$ frequencies). Higher values (i.e., yellow color) indicate highly informative features while blue colors indicate less discriminant features. The scores were normalized for each subject using minmax scaling.

\section{Asynchronous classification}

For online session, we trained, for each subject, a decoder such as described in Section II.F. Fig. 4 reports the features that were selected for the online decoders. Most of the features were located in the upper $\mu$ band.

Fig. 5A shows the latency for each subject during the online session when using our integrative framework for increasing the reliability of BMI outputs (see Section II.C). The latency was computed for every trial by calculating the difference between the time when the participant is supposed to stop and when the clock hand actually stopped moving. On average, we obtained a latency of $-0.1 \mathrm{~s} \pm 1.7 \mathrm{~s}$ (median \pm diff. percentile).

Fig. 5B reports the number of trials where MI termination was detected -on average, in $85.44 \%$ of the trials. For the remaining trials, the clock hand continued rotating for $10 \mathrm{~s}$ until the end of the trial. Interestingly, Fig.5B illustrates that subjects with a high accuracy $(\geq 80 \%)$ also exhibited a small median latency. These results show that participants were able to control the offset of their MI precisely. More importantly, these results indicate that the latency is consistent over the trials because of a relatively small inter-trial variability in most of the subjects (except subject s8).

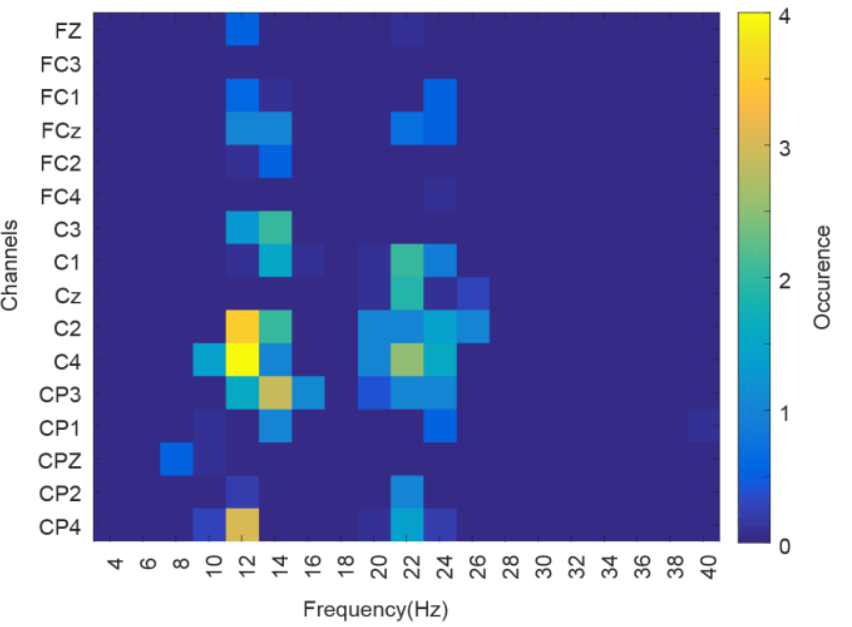

Fig. 4. Feature occurrences for online decoding for all subjects. The number of times a feature was selected for online classifier was counted across subjects. Higher values (i.e., yellow color) indicate highly selected features while blue colors indicate less selected features.

A

B
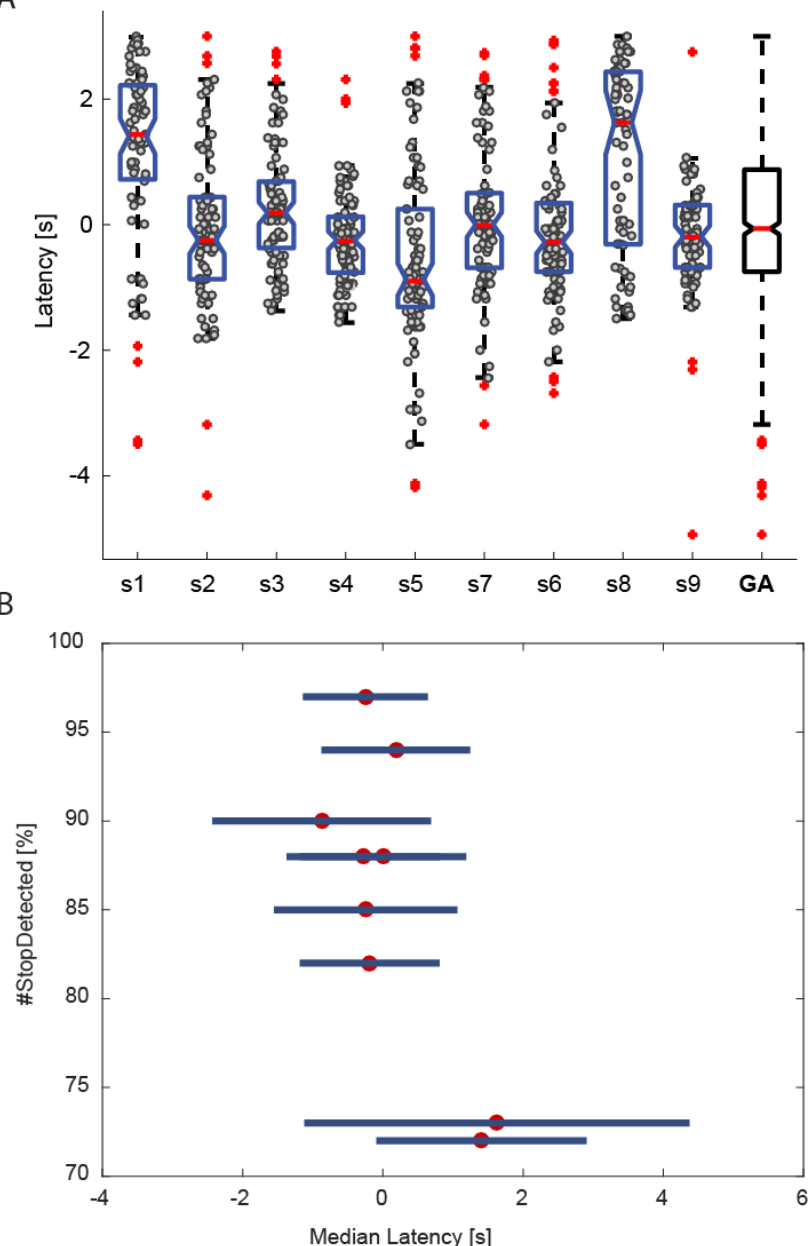

Fig. 5. A. Distribution of latency for every trial and for each subject. Each point corresponds to the latency of one trial and was calculated by computing the difference between the time when the participant is supposed to stop and when the clock hand stopped to move. Boxplots illustrate the distribution and the median latency for every subject. B. The graph shows the median latency in function of the percentage of trials where a stop was detected for each subject. 


\section{Pseudo-online classification}

We further investigated the behavior of our approach, comparing its dynamics between offline and online sessions. To do so, we performed a pseudo-online analysis of the offline session (offline PO), the calibration phase, and each run of the online session (online PO). The results are reported in Fig. 6. On average across all subjects, one can observe that the online PO is shifted back in time, crossing the $54.17 \%$ significance threshold above chance level at $\mathrm{t}=0 \pm 0.11 \mathrm{~s}$ (mean \pm SEM) before offset cue; while the offline PO crosses it at $\mathrm{t}=1.25 \pm$ $0.15 \mathrm{~s}$ (mean \pm SEM) after the offset cue as expected. The calibration phase exhibits a similar behavior to the offline PO. Additionally, we can see that the latency was consistent over online runs. These results confirm that subjects learned rapidly to adapt to the latency of their decoder during closed-loop BMI usage and were able to stop the clock hand precisely on time.

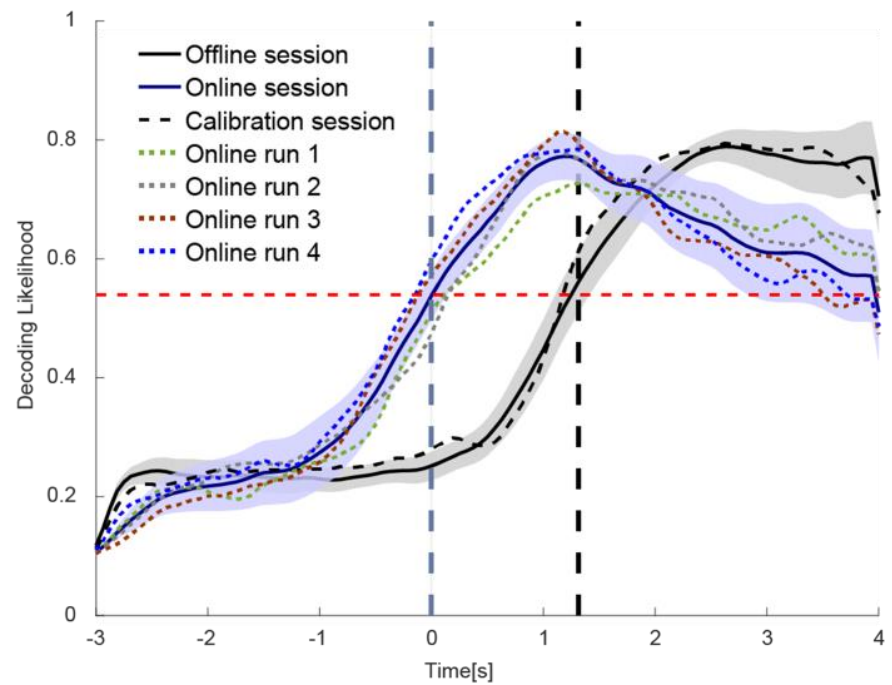

Fig. 6. Pseudo-online analysis and latency of the decoding termination in the offline and online sessions. The figure shows the grand average of decoding likelihood over all subjects in a time interval [-3 4] s with respect to the offset as well as the respective standard error on each time point. The horizontal red line corresponds to the significance threshold above chance level of $54.17 \%$. The filled black line corresponds to the pseudo-online analysis performed on the offline session, while the blue one corresponds to the online session. The dash lines show each individual runs of the online session as well as the calibration phase. The vertical dash lines show where the each pseudo-online is crossing the significance threshold for the offline session and the online session (average over all 4 runs)

\section{E. ERS modulation during online session}

We computed the spectrogram in central channels during the online session (Fig. 7). Compared to the offline session (Fig. $2 \mathrm{~A})$, one can observe a temporal shift of both ERS in the upper $\mu$ and $\beta$ bands that appear now aligned to the offset cue (Fig. 7A). Interestingly, we can also notice stronger ERS patterns, especially in the $\beta$ band. This may be explained by subjects who have more MI practice. Similarly, we evaluated the timing of $\mu$ and $\beta$ ERS $(\alpha=0.05$, repeated measure t-tests based on $t-$ statistics FDR corrected for multiple comparisons). As Fig. 7B illustrates, during the online session, the $\beta$ ERS appeared at -1.1 $\mathrm{s}$; while, as before, the $\mu$ ERS started to develop slightly later at $-0.8 \mathrm{~s}$ with respect to the offset cue. Interestingly, ERS in both bands seemed to last until the end of the trials, as shown by the significant differences w.r.t. the baseline period (top colored bars in Fig 7B). Additionally, we also computed the
A
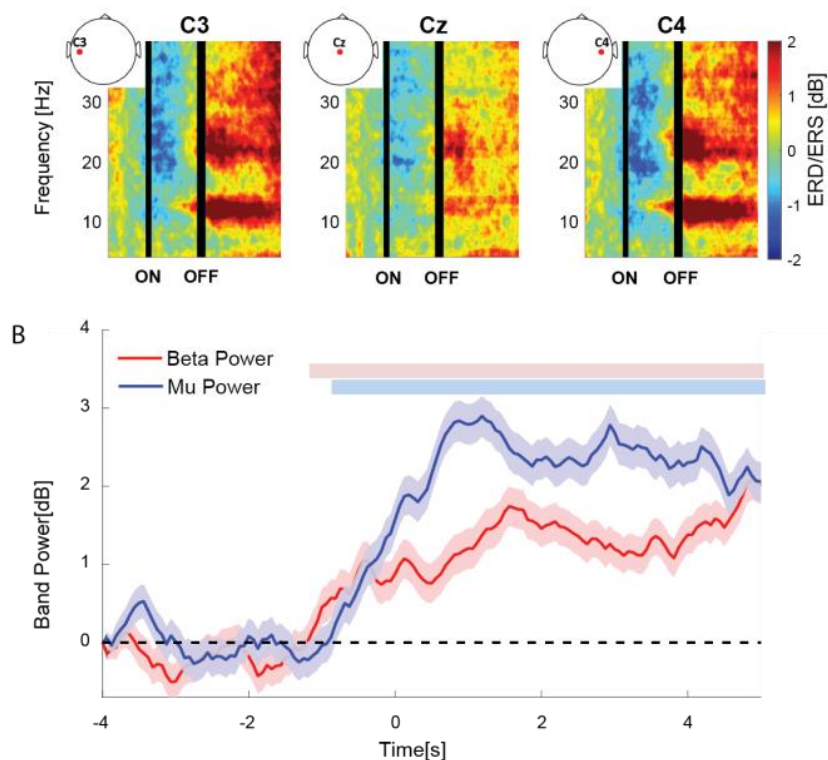

Fig. 7. A. Grand average spectrogram on central channels across subjects and averaged over all trials during the online session. The first vertical line corresponds to the onset $(\mathrm{ON})$ when users initiate their MI, while the second one is the offset (OFF) and corresponds to the time when they stopped MI. The figure was interpolated due to the variation on MI duration. The pre and during MI period [-3 4] s was cropped and joined with the post MI period [0 6] $\mathrm{s}$. ERD/ERS were computed with a logarithmic scale using a baseline period [-2 $-1]$ s. B. Band power of $\mu$ and $\beta$ band averaged over $\mathrm{C} 3$ and $\mathrm{C} 4$ channels for all the trials. The time origin corresponds to the offset. Each color bar on top indicates periods of significant difference with respect to the baseline period ([$3-2] \mathrm{s}$ with respect to the onset) for its corresponding band $(\alpha=0.05$, repeated measure t-tests based on t-statistics, FDR corrected for multiple comparisons).

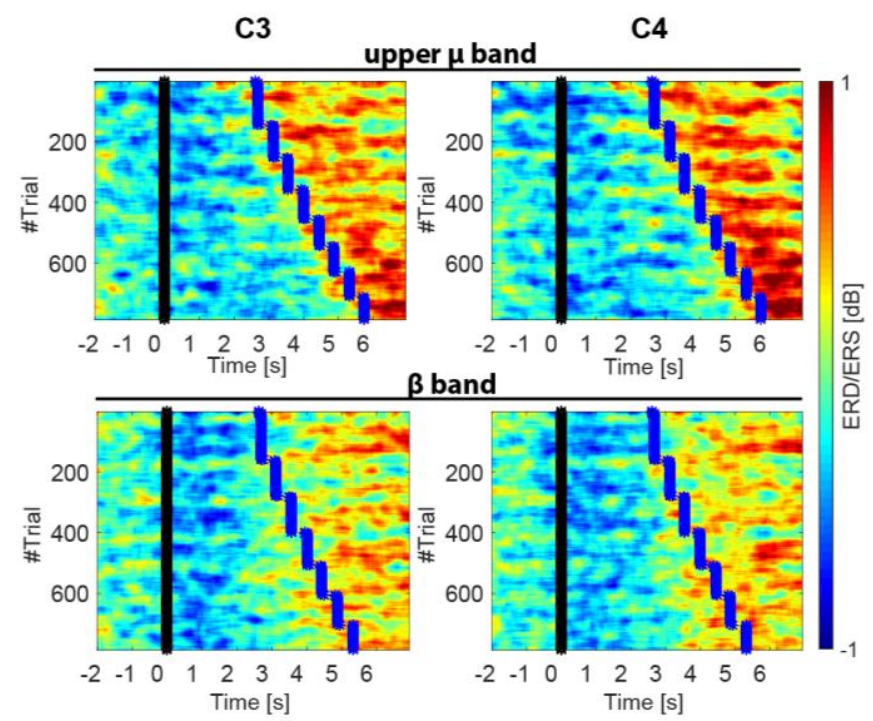

Fig. 8. Average band power of $\mu$ and $\beta$ bands over trials collected from all subjects during the online session for channel $\mathrm{C} 3$ and $\mathrm{C} 4$. All trials including every subject are represented on these heat maps ordered by increasing offset cue time. The first line (black) corresponds to the onset cue where the participant initiates his motor imagery, while the second one (blue) is the offset cue and corresponds to the time when the participant should stop the clock hand. The top panel illustrates, the $\mu$ band, while the bottom panel corresponds to the $\beta$ band

spectrogram for channels $\mathrm{C} 3$ and $\mathrm{C} 4$ at the single trial level (Fig. 8) from every subject in order to investigate the inter-trial variability across the different bands that cannot be observed in the grand average spectrogram. One can notice that the upper $\mu$ 
ERS is well aligned with the offset cue and is consistent over trials. On the other hand, there is a large variability of the $\beta$ rebound over the trials and, more importantly, it is less well aligned with the offset cue.

\section{DISCUSSION}

The present study investigated the feasibility of decoding the spontaneous termination of an imagined movement, based on the natural electrophysiological correlates of such a task. More importantly, we also aimed to answer whether BMI users were able to control the offset of their sensorimotor rhythm in realtime to stop a device accurately at a specific position. We evaluated such an ability by analyzing the BMI performance accuracy and latency.

\section{A. Time-frequency analysis}

From our time-frequency analysis, we observed neural correlates of motor termination consistent with literature [20], [27], [28]. These correlates are characterized by an increase of power in the upper $\mu$ and $\beta$ bands. Comparing these correlates, it appears that the $\mu$ ERS is more prominent and more reliable over subjects since it was observed in eight of them, while significant $\beta$ ERS was found in five subjects. A similar observation has been done in [16] where, after stopping foot MI, $\mu$ ERS was also reported for some subjects.

Moreover, this $\mu$ ERS tends to last longer than $\beta$ ERS, which likely help in detecting MI termination. This difference of duration can be explained by their functional role. Indeed, $\beta$ rebound is identified as a neural correlate of termination [21][23], while $\mu$ rebound is reported as a neural correlate of an idling process [24]-[26]. Because of these different roles, $\beta$ rebound can be seen more as a phasic modulation at offset, whereas $\mu$ synchronization is expected to remain as it is the paradigmatical pattern at rest. Thus, our results are consistent with the current hypotheses regarding the functional roles of both $\beta$ and $\mu$ rhythms [20]-[26]. Importantly, these correlates are found mainly over the sensorimotor cortex (C3 and C4) as well as for some subject in central channel Cz. The location of these correlates is consistent also with fMRI literature for movement termination [15], [33], [34]. Indeed, premotor supplementary area (preSMA) and premotor cortex were identified as brain region involved in the process of stopping a voluntary action. It is also known than the $\beta$ rebound is mainly observed in premotor cortex as well as supplementary motor area [29], [30]. Hence, these results support the putative role of these correlates in the termination of a motor action.

\section{B. Decoding motor imagery termination}

Performance of our decoder were similar to those reported by Bai et al. [17] (average accuracy of $\sim 76 \%$ and $~ 75 \%$, respectively). Nevertheless, and importantly, during closedloop operation, performance of our decoders increased to $\sim 85 \%$ with a negligible latency (see Section IV.C for further discussion). Although our decoder followed the same strategy, the studies mentioned above focused on the $\beta$ band. In contrast, we found that additional information can be extracted from the upper $\mu$ band (see Figs. 3B and 4). Furthermore, the comparison between decoders based on different frequency bands did not show significant differences in term of accuracies. These observations indicate that BMIs should not be based on pre- selected physiological features that may vary from subject to subject, but should be personalized accordingly -e.g., using feature selection on a broader frequency range as done in the present work.

\section{Decoding latency and adaptation}

By looking at the offline PO analysis, we can observe that our decoder shows an average latency close to $1.4 \mathrm{~s}$. This latency can be explained mostly by the neurophysiology of ERS. Indeed, ERS develops around $1 \mathrm{~s}$ after MI offset, which makes an earlier detection implausible. In contrast, the online evaluation (online PO) exhibits decreased average latency with values close to the MI termination cue. We can thus conclude that, based on the BMI feedback provided by the decoder, subjects were able to adapt and compensate the decoding latency, likely by anticipating the right moment to stop MI.

By looking at the spectrograms in the grand average and at the trial level (Figs. 2, 7, and 8), it appears that the neural correlates of MI termination in the upper $\mu$ band are much more consistent between subjects and show a higher inter-trial stability than in the $\beta$ band. This consistency leads to a robust decoding of motor termination and make the responses of the system more predictable. This probably enabled users to stop accurately on target.

On the other hand, $\beta$ modulations seem also to play a critical role during the closed-loop experiments, supporting that the decoders are detecting MI termination and not just the rest state after the end of the task. Although in the offline condition the performance curve of the detector shows a plateau (Fig. 6), indicating that there might be a strong component of rest, this is not the case once subjects go online and learn to anticipate (and eventually modulate) their brain signals. The sharper detection curve for the online sessions (Fig. 6), decaying about $1 \mathrm{~s}$ after $\mathrm{t}=0$, suggests that the classifier is not simply decoding the rest state, but a fast transitory EEG correlate associated to MI termination. This correlate is likely the $\beta$ rebound observed in Figure 7, which is initially very prominent and then decays rapidly. The decoders, which use $\beta$ features (Fig. 4), should be detecting the $\beta$ rebound.

Previous work has shown how to interact and control robots based on asynchronous decoding on MI vs. rest. In particular, Müller-Putz and colleagues [35] asked subjects to sustain MI for 2 different durations, which differed in 2 seconds or more, in order to deliver two different commands. While some subjects could achieve fast transitions for the short-duration commands ( $1 \mathrm{~s})$, this was not the case for long-duration commands. In our case, subjects started with a stop latency of around $1 \mathrm{~s}$ no matter the duration of the MI period. Furthermore, and critically, during closed-loop use subjects in our approach could adapt their self-paced performance to achieve high temporal accuracy. In the discussion of the mentioned paper, authors discuss the difference observed between short and longduration commands by explaining that a MI pattern cannot be actively stopped by the BCI user. On the opposite, our data shows that a BCI user can learn how to stop these patterns if the paradigm and decoder focus explicitly on MI termination. 


\section{CONCLUSION}

We presented an approach to decode the termination of an imagined movement using both the upper $\mu$ and $\beta$ rhythms. To the best of our knowledge, we are showing for the first time that offset MI decoding has an intrinsic latency mainly due to late appearance of neural correlates of motor termination; however, and critically, this latency can be compensated by BMI users. This compensation is only possible because of the reliability of the decoder as well as the consistency of offset correlates, which makes it possible for users to predict their BMI dynamics. This result also highlights the importance of online evaluation of BMI systems and the implications that closedloop interactions have in the system performance [4], [36]. The natural scenario for detection of MI termination is its combination with detection of MI initiation for natural asynchronous control of neuroprostheses. Such an approach would, for instance, allow users to better control the degree of grasping by exploiting two different but associated MI processes, namely MI initiation and termination.

\section{REFERENCES}

[1] J. d. R. Millán et al., "Combining brain-computer interfaces and assistive technologies: State-of-the-art and challenges," Front. Neurosci., vol. 4, p. 161, 2010.

[2] N. Birbaumer et al., "A spelling device for the paralysed," Nature, vol. 398, no. 6725. pp. 297-298, 1999.

[3] S. Perdikis et al., "Clinical evaluation of BrainTree, a motor imagery hybrid BCI speller," J. Neural Eng., vol. 11, no. 3, 2014.

[4] S. Perdikis, L. Tonin, S. Saeedi, C. Schneider, and J. d. R. Millán, "The Cybathlon BCI race: Successful longitudinal mutual learning with two tetraplegic users," PLoS Biol., vol. 16, no. 5, p. e2003787, 2018.

[5] F. Galán et al., "A brain-actuated wheelchair: Asynchronous and noninvasive Brain-computer interfaces for continuous control of robots," Clin. Neurophysiol., vol. 119, no. 9, pp. 2159-2169, 2008.

[6] R. Leeb, L. Tonin, M. Rohm, L. Desideri, T. Carlson, and J. d. R. Millán, "Towards independence: A BCI telepresence robot for people with severe motor disabilities," Proc. IEEE, vol. 103, no. 6, pp. 969982, 2015

[7] G. R. Müller-Putz, R. Scherer, G. Pfurtscheller, and R. Rupp, "EEGbased neuroprosthesis control: A step towards clinical practice," Neurosci. Lett., vol. 382, no. 1-2, pp. 169-174, 2005.

[8] M. Tavella, R. Leeb, R. Rupp, and J. d. R. Millán, "Towards natural non-invasive hand neuroprostheses for daily living," in 2010 Annual International Conference of the IEEE Engineering in Medicine and Biology Society, EMBC'10, 2010, pp. 126-129.

[9] R. Beveridge, S. Wilson, M. Callaghan, and D. Coyle, "Neurogaming with motion-onset visual evoked potentials (mVEPs): adults versus teenagers," IEEE Trans. Neural Syst. Rehabil. Eng., vol. 27, no. 4, pp. 572-581, 2019.

[10] D. Coyle, J. Stow, K. McCreadie, J. McElligott, and Á. Carroll, "Sensorimotor modulation assessment and brain-computer interface training in disorders of consciousness," Arch. Phys. Med. Rehabil., vol. 96, no. 3, pp. S62-S70, 2015.

[11] B. Orset, K. Lee, R. Chavarriaga, and J. d. R. Millán, "Reliable decoding of motor state transitions during imagined movement," 9th Int. IEEE EMBS Conf. Neural Eng., vol. 2019-March, pp. 263-266, 2019.

[12] I. K. Niazi, N. Jiang, and O. Tiberghien, "Detection of movement intention from single-trial movement-related cortical," J. Neural Eng., vol. 66009, no. 8, pp. 1-10, 2011.

[13] E. Lew, R. Chavarriaga, S. Silvoni, and J. d. R. Millán, "Detection of self-paced reaching movement intention from EEG signals," Front. Neuroeng., vol. 5, p. 13, 2012.

[14] M. Schultze-kraft, D. Birman, M. Rusconi, C. Allefeld, K. Görgen, and S. Dähne, "The point of no return in vetoing self-initiated movements," Proc. Natl. Acad. Sci., vol. 113.4, pp. 1080-1085, 2016.
"Selection and stopping in voluntary action: A meta-analysis and combined fMRI study," Neuroimage, vol. 86, pp. 381-391, 2014.

G. Pfurtscheller and T. Solis-Escalante, "Could the beta rebound in the EEG be suitable to realize a "brain switch'?," Clin. Neurophysiol., vol. 120, no. 1, pp. 24-29, 2009.

[17] O. Bai, P. Lin, S. Vorbach, M. K. Floeter, N. Hattori, and M. Hallett, "A high performance sensorimotor beta rhythm-based braincomputer interface associated with human natural motor behavior," J. Neural Eng., vol. 5, no. 1, pp. 24-35, 2008.

[18] B. Libet, C. A. Gleason, E. W. Wright, and D. K. Pearl, "Time of conscious intention to act in relation to onset of cerebral (readinesspotential)," Brain, vol. 106, pp. 623-642, 1983.

[19] J. Pereira, A. I. Sburlea, and G. R. Müller-Putz, "EEG patterns of selfpaced movement imaginations towards externally-cued and internally-selected targets," Sci. Rep., vol. 8, no. 1, 2018.

[20] G. Pfurtscheller, C. Brunner, A. Schlo, and F. H. Lopes Da Silva, "Mu rhythm ( de ) synchronization and EEG single-trial classification of different motor imagery tasks," Neuroimage, vol. 31, pp. 153-159, 2006.

[21] E. Houdayer, E. Labyt, F. Cassim, J. L. Bourriez, and P. Derambure, "Relationship between event-related beta synchronization and afferent inputs: Analysis of finger movement and peripheral nerve stimulations," Clin. Neurophysiol., vol. 117, no. 3, pp. 628-636, 2006.

[22] F. Cassim et al., "Does post-movement beta synchronization reflect an idling motor cortex?," Neuroreport, vol. 12, no. 17, pp. 38593863,2001

[23] A. K. Engel and P. Fries, "Beta-band oscillations-signalling the status quo?," Curr. Opin. Neurobiol., vol. 20, no. 2, pp. 156-165, 2010. G. Pfurtscheller, "Event-related synchronization (ERS): an electrophysiological correlate of cortical areas at rest," Electroencephalogr. Clin. Neurophysiol., vol. 83, no. 1, pp. 62-69, 1992

[25] G. Pfurtscheller and C. Neuper, "Event-related synchronization of mu rhythm in the EEG over the cortical hand area in man," Neurosci. Lett., vol. 174, no. 1, pp. 93-96, 1994.

[26] G. Pfurtscheller, A. Stancák, and C. Neuper, "Event-related synchronization (ERS) in the alpha band - An electrophysiological correlate of cortical idling: A review," Int. J. Psychophysiol., vol. 24, no. 1-2, pp. 39-46, 1996.

[27] G. Pfurtscheller and F. H. Lopes Da Silva, "Event-related EEG/MEG synchronization and desynchronization: Basic principles," Clinical Neurophysiology, vol. 110, no. 11. pp. 1842-1857, 1999.

[28] G. Pfurtscheller, "Functional brain imaging based on ERD/ERS," in Vision Research, 2001, vol. 41, no. 10-11, pp. 1257-1260.

[29] G. Pfurtscheller, C. Neuper, K. Pichler-Zalaudek, G. Edlinger, and F. H. Lopes Da Silva, "Do brain oscillations of different frequencies indicate interaction between cortical areas in humans?," Neurosci. Lett., vol. 286, no. 1, pp. 66-68, 2000.

[30] G. Pfurtscheller, M. Woertz, G. Supp, and F. H. Lopes Da Silva, "Early onset of post-movement beta electroencephalogram synchronization in the supplementary motor area during self-paced finger movement in man," Neurosci. Lett., vol. 339, no. 2, pp. 111114, 2003.

[31] S. Perdikis, H. Bayati, R. Leeb, and J. d. R. Millán, "Evidence accumulation in asynchronous BCI," Int. J. Bioelectromagn., vol. 13, no. 3, pp. 131-132, 2011

[32] S. Makeig, "Auditory event-related dynamics of the EEG spectrum and effects of exposure to tones," Electroencephalogr. Clin. Neurophysiol., vol. 86, no. 4, pp. 283-293, 1993.

[33] D. Swick, V. Ashley, and U. Turken, "Are the neural correlates of stopping and not going identical? Quantitative meta-analysis of two response inhibition tasks," Neuroimage, vol. 56, no. 3, pp. 16551665,2011

[34] J. P. Coxon, A. Van Impe, N. Wenderoth, and S. P. Swinnen, “Aging and Inhibitory Control of Action: Cortico-Subthalamic Connection Strength Predicts Stopping Performance," J. Neurosci., vol. 32, no. 24, pp. 8401-8412, 2012

[35] G. R. Müller-Putz, R. Scherer, G. Pfurtscheller, and C. Neuper, "Temporal coding of brain patterns for direct limb control in humans," Front. Neurosci., vol. 4, no. JUN, 2010.

[36] R. Chavarriaga, M. Fried-Oken, S. Kleih, F. Lotte, and R. Scherer, "Heading for new shores! Overcoming pitfalls in BCI design," BrainComputer Interfaces, vol. 4, no. 1-2, pp. 60-73, 2017. 